\title{
Destabilized green fluorescent protein detects rapid removal of transcription blocks after genotoxic exposure
}

\author{
Nataliya Kitsera, Andriy Khobta, and Bernd Epe
}

BioTechniques 43:222-227 (August 2007)

doi 10.2144/000112479

\begin{abstract}
High stabilities of reporter proteins and their messenger RNAs (mRNAs) interfere with the detection of rapid transient changes in gene expression, such as transcriptional blocks posed by genotoxic DNA lesions. We have modified a green fluorescent protein (GFP) gene within the episomal pMARS vector by addition of a fragment encoding for mouse ornithine decarboxylase (ODC) proline-glutamate-serine-threonine-rich (PEST) sequence in order to target the protein to the proteasomes and achieved an unprecedentedly fast GFP turnover in permanently transfected human cells. As early as $1 \mathrm{~h}$ after inhibition of protein synthesis by cycloheximide, the number of fluorescent cells decreased more than 5-fold. Concordantly, treatments with transcription inhibitors $\alpha$-amanitin and 5,6-dichloro-1- $\beta$-D-ribofuranosylbenzimidazole (DRB) resulted in progressive depletion of the destabilized GFP, detected as fluorescence decline, while the stable protein levels were not affected under the same conditions. Moreover, fluorescence of the destabilized but not of normal GFP decreased strongly and in a dose-dependent manner following an instant transcription block induced by ultraviolet-C (UVC) irradiation. In agreement with the transient nature of the transcriptional block due to transcription-coupled DNA repair, the GFP fluorescence fully recovered after several hours.
\end{abstract}

\section{INTRODUCTION}

Cessation of transcription occurs very rapidly when genotoxic lesions that act as roadblock for RNA polymerases are induced in the template DNA strand (1-4). This transcription block is physiologically important since it triggers a specialized transcription-coupled DNA repair $(\mathrm{TCR})$ pathway $(5,6)$. Hence the mechanism underlying the establishment of the block and its subsequent release are intensively studied at the present. Reporter proteins can be highly useful to monitor the generation and processing of the transcription-blocking DNA modifications in specific genes. Green fluorescent protein (GFP) is a widely used reporter for gene expression studies because of its bright and stable fluorophore, low toxicity, and ease of quantitative detection without disrupting the cell (7). Since GFP is very stable in cells (8), it accumulates to high levels that facilitate detection. At the same time, this high stability makes the protein unsuitable for the tasks when the dynamical changes in gene expression are to be studied.

To overcome this problem, some destabilized variants of fluorescent proteins have been engineered during the last decade. Different groups succeeded in considerably reducing the protein lifetimes by the fusion of natural protein degradation signals. The most promising results were obtained with the use of an ornithine decarboxylase (ODC) prolineglutamate-serine-threonine-rich (PEST) sequence fused to the $\mathrm{C}$ terminus of the reporter proteins. The PEST domain is well-known to be responsible for fast proteasomal degradation of ODC in many animal species $(9,10)$ and commits rapid turnover when transferred to naturally stable proteins (11). Wild-type GFP has a half-life of about $26 \mathrm{~h}$. However, when PEST-containing sequences from mouse $O d c$ gene were fused to GFP, the protein half-life shortened to $5.5 \mathrm{~h}$ in permanent transfection experiments (8) and even $2 \mathrm{~h}$ (12) in transiently transfected cells. To accelerate turnover, these proteins were further destabilized with a mouse cyclin $\mathrm{B}$ destruction box in addition to the ODC fragment (8) or by engineering of the PEST sequence optimized for the most efficient degradation (12). Firefly luciferase is intrinsically less stable than GFP and its half-life could be further reduced to somewhat shorter than $1 \mathrm{~h}$ by a similar fusion approach (13). Yet, even when a reporter protein with a sufficiently high clearance rate is used, the stability of its messenger RNA (mRNA) might still interfere with sensitive and fast detection of changes in gene expression. Indeed, improved responses to a transcriptional repressor were achieved with doubledestabilized reporter constructs that contained both protein and RNA decay determinants $(14,15)$. 

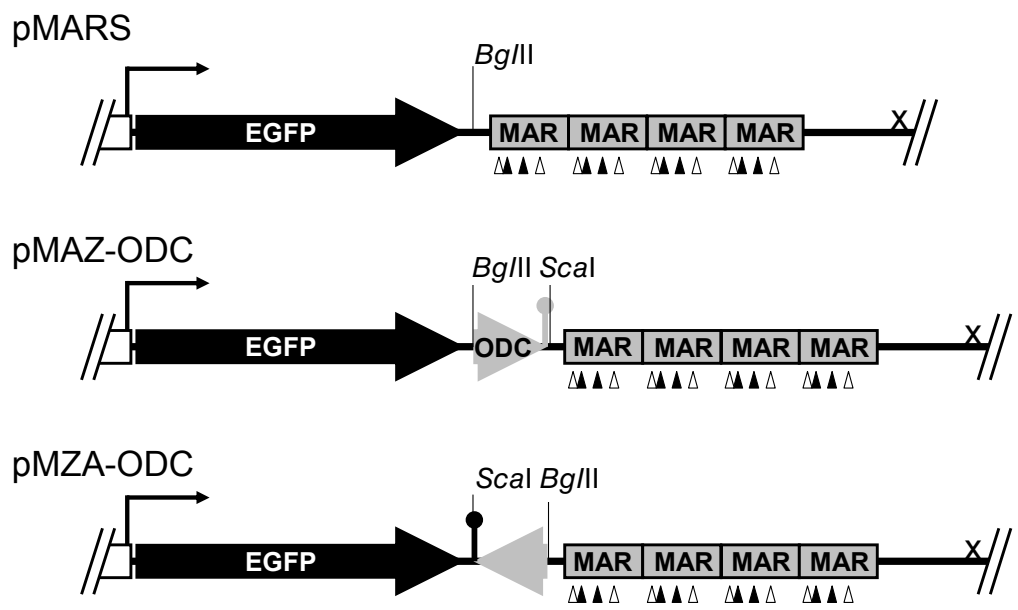

Figure 1. Representation of the green fluorescent protein (GFP) gene constructs used in this study. pMAZ-ODC (central) and pMZA-ODC (bottom) accommodate the ornithine decarboxylase (ODC) gene fragment inserted in opposing orientatations into the $B g l I I$ site of pMARS plasmid (top). Broken arrow indicates transcription start. A fragment of an open box shows the promoter region, bold arrows stand for coding regions of GFP (black) and ODC (gray). Gray lollipop marks the retained original ODC translation stop codon in pMAZ-ODC. Black lollipop stands for the newly inserted stop codon in pMZA-ODC. The constructs share the common $3^{\prime}$ untranslated region (UTR) from pMARS with the shown transcripts' $3^{\prime}$ ends (crosses) and four minimal matrix attachment region (MAR) elements from the human $\beta$-interferon gene (gray boxes). Each of the MAR elements contains two ATTTA (black arrowheads) and two TTATTT (white-filled arrowheads) motifs.

Here, we used an episomal expression vector carrying a strong constitutive promoter and the signal elements for a rapid decay of GFP mRNA and further destabilized the protein by fusion with a mouse ODC fragment. The goal of this work was to create stably transfected cell lines in which reporter protein expression could be monitored in nearly realtime without harmful effects on the host cells. We describe a sufficiently sensitive reporter system to allow the detection of the short-lasting transcription block caused by ultraviolet-C (UVC) in mammalian cells.

\section{MATERIALS AND METHODS}

\section{Vectors}

pMARS episomal vector (16) contains a modified GFP gene from Aequorea victoria (17) that is expressed under the control of the human cytomegalovirus (CMV) promoter and harbors in its 3' untranslated region (UTR) a nuclear scaffold/matrix attachment region (MAR) core element from the human interferon gene repeated four times. pMARS vector was linearized by using an available $B g l I I$ site between the GFP and MAR sequences, and the produced overhangs were filled in with T4 DNA polymerase (New England Biolabs, Frankfurt am Main, Germany) for a subsequent bluntend cloning of the ODC fragment. The 3' portion of $O d c$ gene exon 10 (Mouse Genome Informatics; accession no. MGI:97402) was produced from mouse genomic DNA by PCR using PfuTurbo ${ }^{\circledR}$ DNA Polymerase (Stratagene, Amsterdam, The Netherlands). 5'Phosphorylated primers (Operon, Cologne, Germany) were the following: 5'-TCTCATGAAGCAGATCCAGA-3' (forward) and 5'-TAGTACTCATCTA CACATTGATCCTAG-3' (reverse) and amplified the fragment encoding for the $46 \mathrm{C}$-terminal amino acid residues of ODC. Underlined portions of the oligonucleotides correspond to $O d c$ gene sequence, and bold letters correspond to stop-codons for the two possible insert orientations. PCR products were purified from the agarose gel and ligated into the linearized blunted vector in random orientations. To pick the clones containing the ODC fragment in sense (designated AZ) and antisense (ZA) directions, 30 clones were screened by three-primer PCR with primers: 5'-GACCACTACCAGCAGAACAC$3^{\prime}$ (GFP), 5'-TCTCCTGGGCACAA GACAT-3' (AZ-ODC), and 5'-GCCTG
TGCTTCTGCTAGGAT-3' (ZA-ODC). After sequencing of selected positive clones, we named them pMAZ-ODC (for the correct in-frame insertion of ODC fragment) and pMZA-ODC (for the inverted ODC fragment, preceded by the PCR-introduced translation stopcodon).

\section{Cell Lines and Clones}

HeLa cells were propagated in Dulbecco's modified Eagle's medium (DMEM) supplemented with 10\% fetal calf serum, $2 \mathrm{mM}$ sodium pyruvate, 100 $\mathrm{U} / \mathrm{mL}$ penicillin, and $100 \mathrm{mg} / \mathrm{L}$ streptomycin (all from PAA Laboratories GmbH, Pasching, Austria). Cells were transfected with 800 ng supercoiled pMAZ-ODC and pMZA-ODC plasmids in 6-well plates with the help of Effectene ${ }^{\circledR}$ (Qiagen, Hilden, Germany). Cells were left for 24-48 h after transfections, then collected, and those expressing GFP were separated by fluorescence-activated cell sorting (FACS) using a BD FACSVantage ${ }^{\mathrm{TM}}$ cell sorter (Becton Dickinson, $\mathrm{GmbH}$, Heidelberg, Germany) and plated in the selection medium containing $800 \mathrm{mg} / \mathrm{L}$ G418. Single fluorescent clones were identified under a microscope, picked, and expanded under the selection pressure for 4 weeks.

\section{Cell Treatments}

$\alpha$-amanitin was dissolved in sterile water, and all other inhibitors in dimethyl sulfoxide (DMSO). Final concentration of DMSO during the treatments was $0.1 \%$, which by itself had no effect on GFP fluorescence (data not shown). All inhibitors were from Sigma-Aldrich (Taufkirchen, Germany). Appropriate vehicle controls were always included. UVC irradiation was performed in uncovered 6-well culture plates on a black background with a $15 \mathrm{~W}$ mercury low-pressure lamp at the dose intensity $0.3 \mathrm{~W} / \mathrm{m}^{2}$ as measured with a Luxmeter 100 dosimeter (PRC Krochmann, Berlin, Germany). Medium was removed from cells for the time of irradiation. For every irradiated cell sample, a darkincubated sample was processed in parallel and used for normalization. The fluorescence of the UVC-irradiated cells 


\section{Research Reports}

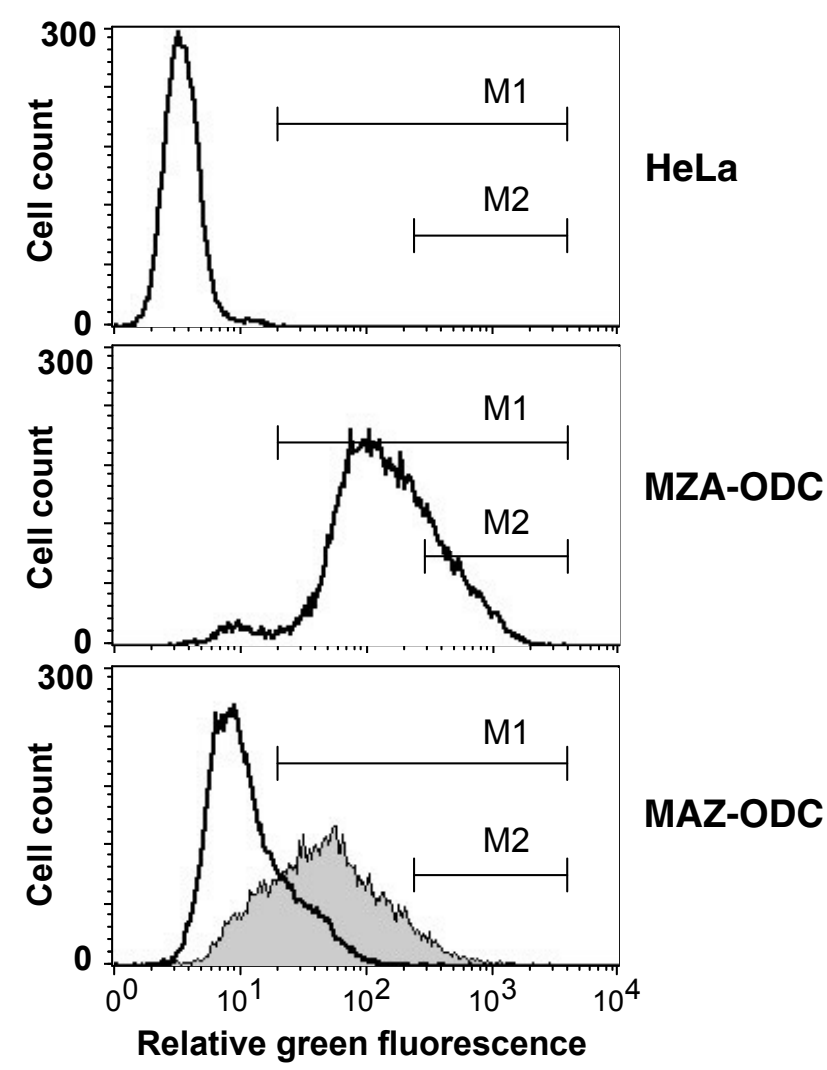

Figure 2. Green fluorescent protein (GFP) fluorescence in HeLa cells stably transfected with pMZA-ODC (unmodified GFP) and pMAZ-ODC (destabilized GFP) constructs. Flow cytometric analyses of wild-type HeLa cells (top panel) and single MZA-ODC (middle) and MAZ-ODC (bottom) clones. Gray overlay in the bottom histogram represents the cells treated with $2 \mu \mathrm{M}$ MG115. Highly fluorescent cells were counted through the regions M1 (MAZ-ODC) and M2 (MZA-ODC) for the following analyses. ODC, ornithine decarboxylase.

was expressed as fraction of the correspondent dark control.

\section{Flow Cytometry}

GFP fluorescence in cells was measured by flow cytometry (FACSCalibur). Cells were formaldehydefixed prior to analyses to avoid any uncontrolled GFP degradation during the sample processing or storage. To this end, cells were detached with trypsin, disaggregated by vigorous mixing with cold medium, washed once with ice-cold Dulbecco's phosphate-buffered saline (DPBS; PAA Laboratories), resuspended in DPBS at $1-2 \times 10^{6} \mathrm{~mL}^{-1}$, instantly mixed with equal volume of cold $2 \%$ formaldehyde prepared in DPBS, and allowed to fix 12$24 \mathrm{~h}$ in the refrigerator. All centrifugation steps were at $250 \times g$. On the next day, the fixed cells were washed with DPBS and analyzed. The fixation procedure had no detectable effect on GFP fluorescence. The FACS instrument was calibrated by the nontransfected HeLa cells. Due to the rapid turnover of destabilized GFP, the majority of cells in pMAZ-transfected clones exhibited very low fluorescence. Therefore, the percentages of highly fluorescent cells, rather than mean fluorescence values, were measured as previously described (12). The fluorescence threshold was arbitrarily chosen to cut off $99.9 \%$ of nontransfected cells (see Results section).

\section{RESULTS}

\section{Molecular Constructs}

We choose the episomal pMARS vector (16) for expression of a destabi- lized GFP for the following reasons: $(i)$ this vector is replicated and maintained at a stable number of copies in a variety of mammalian cell lines (16); (ii) the transgene expression cannot be influenced by its integration site in the chromosomal DNA since the vector propagates as an episome (18); and (iii) the strong CMV promoter should ensure sufficient mRNA levels and allow high-detection sensitivity even for a double-destabilized reporter. Finally, the modified GFP transcript from pMARS plasmid contains an AUrich $3^{\prime}$-UTR due to transcription of the MAR sequences (16). From the DNA sequence, we identified two classes of AU-rich elements (ARE) that are known to promote a rapid decay of variety of human mRNAs $(19,20)$. So, the AUUUA pentanucleotide that constitutes class-I ARE is present twice within each of the four MAR elements, (i.e., in eight copies in the 3'-UTR of GFP mRNA) (Figure 1). The same is true for the TTATTT motif that resembles a class-II ARE. In accordance with these observations, the cells transfected with pMARS plasmid had lower levels of GFP expression than those transfected with the equal amounts of pEGFP-C3 vector that had no ARE in the transcript's 3'-UTR (data not shown).

We have constructed two related vectors by cloning the mouse $O d c$ gene fragment in two directions into the BglII site of pMARS. The vector named pMAZ-ODC was designed to provide correct translation of the ODC fragment joined via an SGLRS peptide to the GFP $\mathrm{C}$ terminus, so that the whole protein would be targeted to rapid degradation in proteasome. The other vector named pMZA-ODC encoded for stable GFP due to a translation stop-codon introduced before the inverted ODC sequence (Figure 1). BglII sites were reconstituted at the $5^{\prime}$ end of ODC coding sequence and converted to $S c a$ I sites on the ODC $3^{\prime}$ side by the PCR design, with total insert lengths of $157 \mathrm{bp}$ in each plasmid. Thus, pMAZ-ODC and pMZA-ODC vectors have the same size of $5742 \mathrm{bp}$ and have the same size, direction, and base content of the transcribed regions, as well as the entire $3^{\prime}$ portion of the of GFP transcripts; the ODC insert orientation is the only difference between these plasmids at the molecular level (Figure 1). 


\section{Research Reports}

A
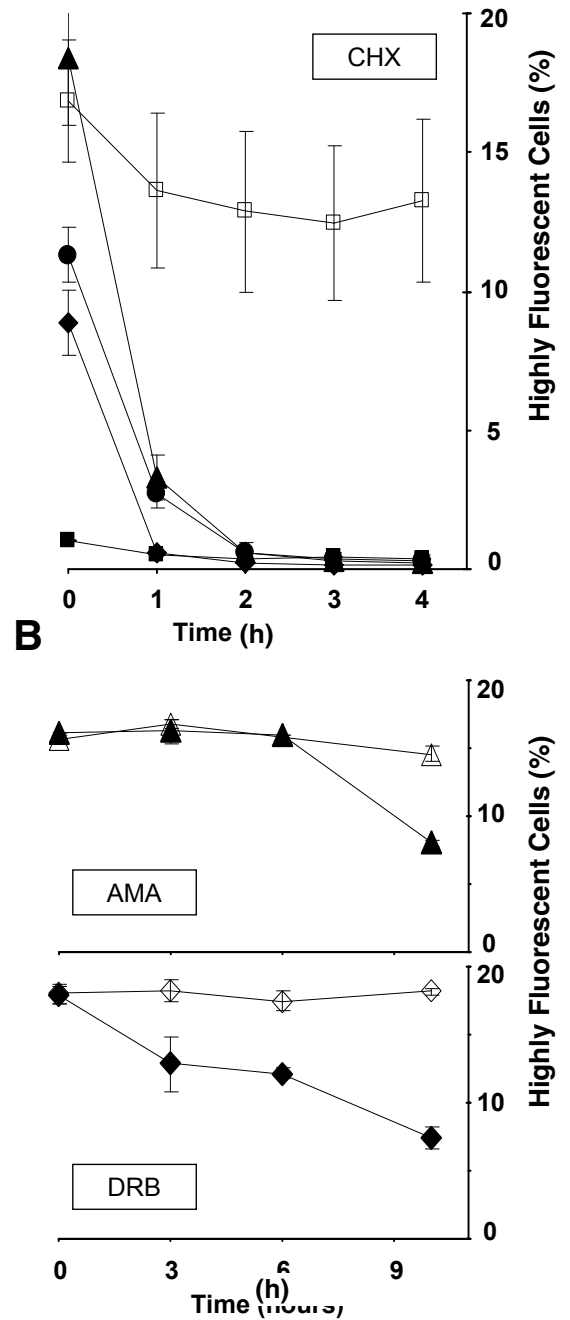

Figure 3. Fluorescence of normal and destabilized green fluorescent protein (GFP) after inhibition of protein or messenger RNA (mRNA) synthesis in stably transfected HeLa cells. (A) Kinetics of GFP degradation measured as fluorescence decline after addition of $100 \mu \mathrm{M}$ cycloheximide (CHX). Open symbols, unmodified GFP (a representative MZA-ODC clone); closed symbols, destabilized GFP (four different MAZODC clones). (B) GFP fluorescence after transcription inhibition by $10 \mu \mathrm{M} \alpha$-amanitin (AMA; upper panel) and $20 \mu \mathrm{M}$ 5,6-dichloro-1- $\beta$-Dribofuranosylbenzimidazole (DRB; lower panel). Open symbols, unmodified GFP (MZA-ODC); closed symbols, destabilized GFP (MAZ-ODC). Data in panels A and B indicate percentages of highly fluorescent cells. Mean of two or three independent experiments \pm SD. ODC, ornithine decarboxylase.

\section{Characterization of Permanently Transfected Clones}

We analyzed six HeLa clones expressing the stable GFP protein from the pMZA-ODC vector. Five of them had green fluorescence similar to the clone shown in Figure 2 (center panel), and one had weaker fluorescence that was, however, clearly distinguishable from the nontransfected cells (data not shown). Cells transfected with pMAZODC vector generally demonstrated much weaker fluorescence. This was attributed to rapid degradation of GFPODC fusion protein in the proteasome, since fluorescence could be saved by treatment with proteasome inhibitor MG115 (Figure 2, bottom panel). MG115 treatment was therefore used to pick the stably transfected clones that retained the GFP construct. Regretfully, in the absence of proteasome inhibition, most of the selected clones showed very low green fluorescence or were not fluorescent at all. Of 25 screened clones, only three showed distinct fluorescence levels when compared with nontransfected cells. In the selected clones, green fluorescence was stably maintained for at least 45 rounds of cell division after the removal of selection pressure (data not shown). Longer times were not tested.

\section{Comparative Stability Analyses of the Reporter Proteins}

To measure the degradation rate of destabilized GFP, we inhibited protein synthesis in the cells with cycloheximide. All three pMAZ-ODC-transfected clones that initially had sizeable GFP levels now demonstrated rapid kinetics of GFP decay manifested as drastic reduction of GFP fluorescence already within the first hour. The fluorescence disappeared completely between 1 and $2 \mathrm{~h}$ of cycloheximide treatment (Figure 3A). A similarly rapid GFP degradation seemed to occur in other MAZ-ODC clones, although this could not be so clearly documented due to the low-fluorescence signal of these cells (a clone is shown with closed squares in Figure 3A).

When the same fluorescence threshold was applied to MZA-ODC cells that express GFP with unaltered stability, we reproducibly found $>97 \%$ cells with high fluorescence levels (Figure 2). Cycloheximide neither affected the percentage of fluorescent cells, nor did it induce any noteworthy decrease in their mean fluorescence intensity (data not shown). To exclude any possible artifact due to the threshold selection, we then applied an equivalent threshold fluorescence level to the MZA-ODC cell line (note the left margin of the region M2 in Figure 2). It delimited $16 \%-20 \%$ of the brightest fluorescent cells, as in case of the MAZ-ODC cell line. Such analysis setup enabled a direct comparison between the cell lines (Figure 3). As previously described, we did not detect any significant decay of the stable GFP protein (no ODC fusion) over $4 \mathrm{~h}$ of cycloheximide treatment (Figure 3A).

Treatment of the cells with a specific RNA polymerase II inhibitor $\alpha$-amanitin also revealed big differences between the normal and destabilized GFP proteins (Figure 3B). In this case, fluorescence decline was much slower than with the protein synthesis inhibitor cycloheximide, possibly due to a slow cellular uptake of $\alpha$-amanitin (21). To verify that delayed response to $\alpha$-amanitin was due to the poor uptake and not due to a slow mRNA degradation, we treated the cells with another transcription inhibitor, DRB. Fluorescence of destabilized GFP decreased already after $3 \mathrm{~h}$ of treatment and continued to descend till the 10-h time point. In contrast, no decrease at all was registered in MZA-ODC cells expressing the stable GFP (Figure 3B).

\section{Application of Destabilized GFP to Detect Transient Transcriptional Block Posed by DNA Damage}

To further analyze the effect of transcriptional block on the expression of destabilized GFP, we induced DNA photo-adducts by UVC irradiation of the cells. These UV lesions mostly consist of cyclobutane pyrimidine dimers (CPD) with some contribution of 6-4 photoproducts $(22,23)$. Both lesion types efficiently block transcription via RNA polymerase arrest and premature termination of RNA synthesis at the lesion sites $(1,2)$. We compared the fluorescence of normal and destabilized GFP over 6 $\mathrm{h}$ after the cell exposure to $254 \mathrm{~nm}$ UV light. The applied UV dose $\left(140 \mathrm{~J} / \mathrm{m}^{2}\right)$ is calculated to induce on average 2.4 CPD per thousand base pairs in chromosomal DNA $(24,25)$. Under these irradiation conditions, $90 \%$ of the template strands of the GFP genes are expected to contain CPD. Despite such a vast 


\section{Research Reports}

A

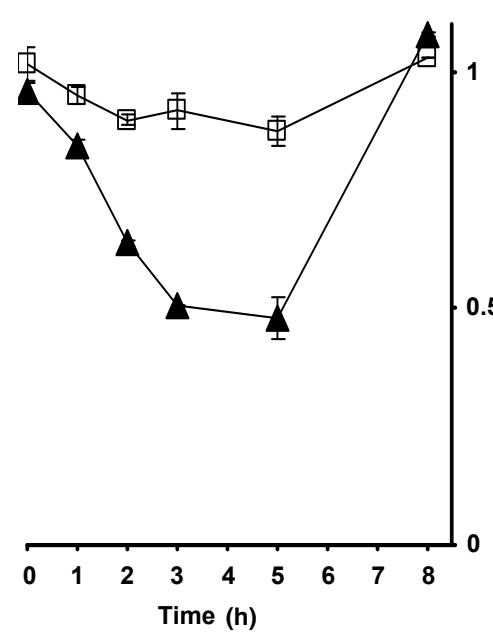

B

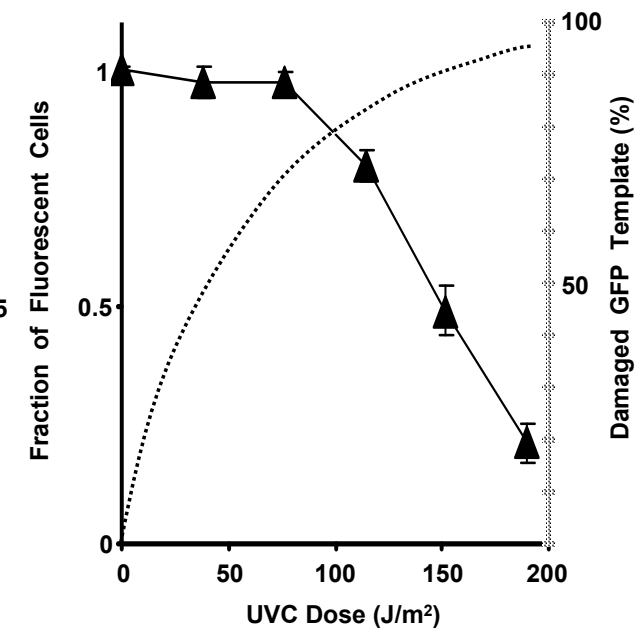

Figure 4. Green fluorescent protein (GFP) fluorescence following the transcription block induced by $254 \mathrm{~nm}$ ultraviolet-C (UVC) irradiation. The transcription-blocking DNA adducts were induced in stably transfected cells by UVC. Fluorescence values are expressed as fractions of the correspondent dark controls. (Left panel) Comparison between the fluorescence of unmodified (MZA-ODC cells, open squares) and destabilized (MAZ-ODC cells, closed triangles) GFP at various time points after irradiation with $140 \mathrm{~J} / \mathrm{m}^{2} \mathrm{UVC}$. Mean values of two independently processed cell sample series $\pm \mathrm{SD}$. (Right panel) effect of various UV doses on the fluorescence of destabilized GFP $2.5 \mathrm{~h}$ after irradiation. Mean of three independently processed sample series \pm SD. Dotted line traces the probability of transcription-blocking lesion to occur within the transcribed DNA strand of GFP-Odc gene. ODC, ornithine decarboxylase.

load of transcription-blocking lesions, fluorescence of the unmodified GFP, measured in MZA-ODC cells, decreased very slightly, compared with the nonirradiated cells. In contrast, destabilized GFP showed a much more pronounced fluorescence decline after UVC irradiation. This was documented as a $50 \%$ decrease in the fraction of fluorescent MAZ-ODC cells between 3 and $4 \mathrm{~h}$ following the irradiation (Figure 4). In the case of destabilized GFP, a very small (about 4\%) decrease of fluorescence was already observed directly after irradiation (i.e., within the sample processing time of 15-20 min. Interestingly, full fluorescence recovery was achieved for both proteins by $6 \mathrm{~h}$ post-UV irradiation).

Finally, we used a range of UV doses to correlate the extent of fluorescence loss measured $2.5 \mathrm{~h}$ after the damage induction with the extent of damage within the transcribed region of the destabilized GFP gene. The results show that the residual GFP fluorescence is negatively correlated with the calculated probability for the transcribed DNA strand of the GFP gene (1870 bases) to contain a transcription-blocking UV-photoadduct (Figure 4). Similar dose-responses were observed under the conditions of transient transfection, although the initial GFP fluorescence was highly variable in function of transfection efficiencies (data not shown). We therefore conclude that destabilized GFP provides a useful tool to detect short-lasting transcription perturbations and transient transcription arrests with sufficient sensitivity.

\section{DISCUSSION}

It is often useful to monitor changes in gene expression in the living cells. The commonly utilized fluorescent proteins such as GFP can serve as reliable reporters in gene induction studies. However, due to the slow degradation rates, their application to detect cessation and resumption of transcription is limited to long-lasting effects. Rapid declines in gene transcription can be of interest when they occur in response to physiological regulatory signals (reviewed in Reference 26) or are caused by genotoxic substances and environmental agents that generate bulky adducts in DNA. These DNA lesions can physically block progression of the RNA polymerases $(1,2)$. Such transcription halt has at least double biological significance. First, it appears responsible for the cytotoxic effects of the bulky DNA lesions (including the checkpoint activation and induction of apoptosis) $(27,28)$. Second, it signals for the active removal of the DNA lesions in a specially evolved transcription-coupled repair pathway $(5,6,29)$.

The double-destabilized GFP constructed and used in this study has a markedly improved performance as a gene expression reporter, compared with a canonical fluorescent protein. This allowed us to detect a short-lasting block of transcription upon the irradiation of cells with UVC, although, due to the small gene size, we had to use highUV doses to produce sufficient density of lesions. To minimize the damage of non-DNA cellular components, we used a single-band 254-nm UV source. Still, we cannot exclude that such damage was generated to some extent and might have interfered with protein synthesis, folding, stability, and fluorofore structure. However, no substantial bleaching or degradation of stable GFP were detected under the same experimental conditions. Furthermore, we did not only observe the GFP fluorescence decrease early following the damage induction, but also its subsequent recovery to the initial level. Remarkably, recovery of the GFP fluorescence occurred in the similar timescale as transcription resumption measured in various cells in course of DNA repair (30-32). Taken together with the dose-response studies (Figure 4B), these findings indicate that the observed changes in the destabilized GFP fluorescence closely correlated with DNA damage generation and repair.

We conclude that the used doubledestabilized reporter can offer several advantages over traditional approaches for measurement of transcriptional activity such as incorporation of labeled uridine. First, it can be used in gene- and promoter-specific applications. Second, gene expression can be easily analyzed in living cells. Third, the renowned benefits of fluorescent reporter proteins are combined with fast turnover, thus allowing the monitoring of gene expression in nearly real-time. This advantage of our double-destabilized reporter is most evident in comparison with a canonical stable GFP. It has to be kept in mind, however, that alterations in RNA and protein turnover can potentially lead to the fluorescence changes as well. 
Although this can pose certain limitation for the use of our reporter system in transcription studies, it can also turn useful for potential application in mRNA and protein metabolism research.

\section{ACKNOWLEDGMENTS}

We are grateful to Dr. Hans Joachim Lipps and Dr. Isa Maria Stehle (University of Witten/Herdecke) for the provided pMARS vector. We thank Heike Arnold and Christian Wiegand for their participation in the cell culture experiments, and Dr. Steffen Schmitt (University of Mainz) for helpful discussions and for the valuable assistance with FACS analyses and cell sorting. The work was supported by the grants from the Deutsche Forschungsgemeinschaft (DFG; no. EP11/5-2) and from the European Commission (CLUSTOXDNA no. MCRTN-CT-2003-505086).

\section{COMPETING INTERESTS STATEMENT}

The authors declare no competing interests.

\section{REFERENCES}

1. Mei Kwei, J.S., I. Kuraoka, K. Horibata, M. Ubukata, E. Kobatake, S. Iwai, H. Handa, and K. Tanaka. 2004. Blockage of RNA polymerase II at a cyclobutane pyrimidine dimer and 6-4 photoproduct. Biochem. Biophys. Res. Commun. 320:1133-1138.

2.Donahue, B.A., S. Yin, J.S. Taylor, D. Reines, and P.C. Hanawalt. 1994. Transcript cleavage by RNA polymerase II arrested by a cyclobutane pyrimidine dimer in the DNA template. Proc. Natl. Acad. Sci. USA 91:8502-8506.

3. Tornaletti, S., B.A. Donahue, D. Reines, and P.C. Hanawalt. 1997. Nucleotide sequence context effect of a cyclobutane pyrimidine dimer upon RNA polymerase II transcription. J. Biol. Chem. 272:31719-31724.

4. Mone, M.J., M. Volker, O. Nikaido, L.H. Mullenders, A.A. van Zeeland, P.J. Verschure, E.M. Manders, and R. van Driel. 2001. Local UV-induced DNA damage in cell nuclei results in local transcription inhibition. EMBO Rep. 2:1013-1017.

5. Mellon, I., G. Spivak, and P.C. Hanawalt. 1987. Selective removal of transcriptionblocking DNA damage from the transcribed strand of the mammalian DHFR gene. Cell $51: 241-249$.
6. Laine, J.P. and J.M. Egly. 2006. Initiation of DNA repair mediated by a stalled RNA polymerase IIO. EMBO J. 25:387-397.

7. Chalfie, M., Y. Tu, G. Euskirchen, W.W. Ward, and D.C. Prasher. 1994. Green fluorescent protein as a marker for gene expression. Science 263:802-805.

8.Corish, P. and C. Tyler-Smith. 1999. Attenuation of green fluorescent protein half-life in mammalian cells. Protein Eng. 12:1035-1040.

9. Russell, D.H. and S.H. Snyder. 1969. Amine synthesis in regenerating rat liver: extremely rapid turnover of ornithine decarboxylase. Mol. Pharmacol. 5:253-262.

10. Rechsteiner, M. and S.W. Rogers. 1996. PEST sequences and regulation by proteolysis. Trends Biochem. Sci. 21:267-271.

11. Loetscher, P., G. Pratt, and M. Rechsteiner. 1991. The C terminus of mouse ornithine decarboxylase confers rapid degradation on dihydrofolate reductase. Support for the PEST hypothesis. J. Biol. Chem. 266:11213-11220.

12.Li, X., X. Zhao, Y. Fang, X. Jiang, T. Duong, C. Fan, C.C. Huang, and S.R. Kain. 1998. Generation of destabilized green fluorescent protein as a transcription reporter. J. Biol. Chem. 273:34970-34975.

13. Leclerc, G.M., F.R. Boockfor, W.J. Faught, and L.S. Frawley. 2000. Development of a destabilized firefly luciferase enzyme for measurement of gene expression. BioTechniques 29:590-601.

14. Voon, D.C., L.S. Subrata, S. Baltic, M.P. Leu, J.M. Whiteway, A. Wong, S.A. Knight, F.T. Christiansen, et al. 2005. Use of mRNA- and protein-destabilizing elements to develop a highly responsive reporter system. Nucleic Acids Res. 33:e27.

15. Benjamin, D., M. Colombi, and C. Moroni. 2004. A GFP-based assay for rapid screening of compounds affecting ARE-dependent mRNA turnover. Nucleic Acids Res. 32:e89.

16. Jenke, A.C., I.M. Stehle, F. Herrmann, T. Eisenberger, A. Baiker, J. Bode, F.O. Fackelmayer, and H.J. Lipps. 2004. Nuclear scaffold/matrix attached region modules linked to a transcription unit are sufficient for replication and maintenance of a mammalian episome. Proc. Natl. Acad. Sci. USA 101:11322-11327

17. Haas, J., E.C. Park, and B. Seed. 1996. Codon usage limitation in the expression of HIV-1 envelope glycoprotein. Curr. Biol. 6:315-324.

18. Lipps, H.J. and J. Bode. 2001. Exploiting chromosomal and viral strategies: the design of safe and efficient non-viral gene transfer systems. Curr. Opin. Mol. Ther. 3:133-141.

19. Chen, C.Y. and A.B. Shyu. 1995. AU-rich elements: characterization and importance in mRNA degradation. Trends Biochem. Sci. 20:465-470.

20. Barreau, C., L. Paillard, and H.B. Osborne. 2005. AU-rich elements and associated factors: are there unifying principles? Nucleic Acids Res. 33:7138-7150.

21. Letschert, K., H. Faulstich, D. Keller, and D. Keppler. 2006. Molecular characterization and inhibition of amanitin uptake into human hepatocytes. Toxicol. Sci. 91:140-149.
22. Cadet, J., E. Sage, and T. Douki. 2005 Ultraviolet radiation-mediated damage to cellular DNA. Mutat. Res. 571:3-17.

23. Yoon, J.H., C.S. Lee, T.R. O'Connor, A. Yasui, and G.P. Pfeifer. 2000. The DNA damage spectrum produced by simulated sunlight. J. Mol. Biol. 299:681-693.

24. Zelle, B., R.J. Reynolds, M.J. Kottenhagen, A. Schuite, and P.H. Lohman. 1980. The influence of the wavelength of ultraviolet radiation on survival, mutation induction and DNA repair in irradiated Chinese hamster cells Mutat. Res. 72:491-509.

25. Kasten, U., D. Beyersmann, J. DahmDaphi, and A. Hartwig. 1995. Sensitive nonradioactive detection of UV-induced cyclobutane pyrimidine dimers in intact mammalian cells. Mutat. Res. 336:143-152.

26. Kadonaga, J.T. 2004. Regulation of RNA polymerase II transcription by sequence-specific DNA binding factors. Cell 116:247-257.

27. Mayne, L.V. and A.R. Lehmann. 1982 Failure of RNA synthesis to recover after UV irradiation: an early defect in cells from individuals with Cockayne's syndrome and xeroderma pigmentosum. Cancer Res. 42:1473-1478.

28. Ljungman, M. and D.P. Lane. 2004 Transcription-guarding the genome by sensing DNA damage. Nat. Rev. Cancer 4:727737.

29. Fousteri, M., W. Vermeulen, A.A. van Zeeland, and L.H. Mullenders. 2006. Cockayne syndrome A and B proteins differentially regulate recruitment of chromatin remodeling and repair factors to stalled RNA polymerase II in vivo. Mol. Cell 23:471-482.

30. Rockx, D.A., R. Mason, A. van Hoffen, M.C. Barton, E. Citterio, D.B. Bregman, A.A. van Zeeland, H. Vrieling, et al. 2000. UV-induced inhibition of transcription involves repression of transcription initiation and phosphorylation of RNA polymerase II. Proc. Natl. Acad. Sci. USA 97:10503-10508.

31. van Hoffen, A., W.H. Kalle, A. de JongVersteeg, A.R. Lehmann, A.A. van Zeeland, and L.H. Mullenders. 1999. Cells from XP-D and XP-D-CS patients exhibit equally inefficient repair of UV-induced damage in transcribed genes but different capacity to recover UV-inhibited transcription. Nucleic Acids Res. 27:2898-2904.

32. McKay, B.C. and M. Ljungman. 1999. Role for p53 in the recovery of transcription and protection against apoptosis induced by ultraviolet light. Neoplasia 1:276-284.

Received 27 January 2007; accepted 19 April 2007.

Address correspondence to Andriy Khobta, Institute of Pharmacy, Johannes Gutenberg University of Mainz, Staudingerweg 5, 55099 Mainz, Germany. e-mail: khobta@uni-mainz.de

To purchase reprints of this article, contact: Reprints@BioTechniques.com 